\title{
Reduced low-frequency heart rate variability relates to greater intimal-medial thickness of the carotid wall in two samples
}

Clara Gautier ${ }^{a}$, Lynn Stine ${ }^{\mathrm{a}}$, J. Richard Jennings ${ }^{\mathrm{a}}$, Kim Sutton-Tyrrell ${ }^{\mathrm{b}}$, Matthew B. Muldoon ${ }^{\text {, }}$ Thomas W. Kamarck ${ }^{a}$, George A. Kaplan ${ }^{d}$, Jukka Salonen ${ }^{\mathrm{e} f}$ and Stephen B. Manuck ${ }^{\mathrm{a}}$

Introduction We investigated the relationship between heart rate variability and preclinical carotid intima-media thickening, a putative index of atherosclerosis.

Methods A sample of $\mathbf{3 5 0}$ men and women (mean age $\mathbf{5 6 . 8}$ years) selected for the presence or absence of untreated hypertension was assessed for heart rate variability at rest and separately for carotid intima-media thickness using duplex ultrasonography (Pittsburgh study). Findings from this sample were cross-validated in a subsample of 68 men drawn from the Kuopio Ischemic Heart Disease Risk Factor trial and selected for the presence or absence of angina.

Results In both samples, regression analyses, controlling for known risk factors, showed a significant negative relationship between mean carotid intima-media thickness and low-frequency $(0.05-0.15 \mathrm{~Hz})$ heart rate variability, but not high-frequency variability.

Discussion The mechanism underlying this relationship remains unclear. The absence of difference in highfrequency variation questions any interpretation in terms of vagal function; the difference in low-frequency variation

\section{Introduction}

Variability in beat-to-beat heart rate has been associated with cardiovascular disease morbidity and mortality. Decreased heart rate variability (HRV) has been observed in persons who have had a myocardial infarction [1,2]. Furthermore, decreased HRV has been associated with sudden cardiac death in chronic heart failure as well as higher mortality risks after myocardial infarction [3-6]. Angiographically diagnosed coronary atherosclerosis and a number of risk factors for coronary artery disease (CAD) are associated with lower HRV [7-12]. These studies led us to ask, first, whether preclinical, less invasive indices of atherosclerosis also relate to reduced $\mathrm{HRV}$; and second, whether reduced $\mathrm{HRV}$ is a marker of early CAD that is independent of known risk factors. If so, HRV may have utility as a predictor of CAD and may contribute to our understanding of CAD pathology. may implicate vessel wall characteristics or decreased sympathetic nervous system influence.

Conclusion Decreased amplitude of low-frequency heart rate variability seems associated with a preclinical atherosclerotic index. Coron Artery Dis 18:97-104 (c) 2007 Lippincott Williams \& Wilkins.

Coronary Artery Disease 2007, 18:97-104

Keywords: atherosclerosis, heart disease, heart rate variability, sympathetic nervous system, vagus

Departments of a Psychiatry and Psychology, ${ }^{b}$ Epidemiology, Graduate School of Public Health, 'Medicine, University of Pittsburgh, Pittsburgh, Pennsylvania, ${ }^{\mathrm{d}}$ Department of Epidemiology and Center for Social Epidemiology and Population Health, University of Michigan, Ann Arbor, Michigan, ${ }^{e}$ Research Institute of Public Health and Department of Community Health and General Practice, University of Kuopio and ${ }^{f} \mathrm{O} y$ Jurilab, Ltd, Kuopio, Finland

Correspondence and requests for reprints to Dr J.R. Jennings, E1329 WPIC, 3811 O'Hara St, Pittsburgh, PA 15213, USA

Tel: +1412 246 6220; fax: + 14122466210 ; e-mail: JenningsJR@upmc.edu

Received 25 January 2006 Revised 22 September 2006 Accepted 25 September 2006
The ultrasound measurement of carotid intima-media thickness (IMT) is an established marker of preclinical atherosclerosis. Carotid IMT has been shown to correlate with angiographic indications of CAD [13,14], with risk factors for CAD $[15,16]$ and prospectively with coronary events, such as infarctions [15,17]. Although IMT has generally been found to associate with coronary atherosclerosis, the findings have been stronger in the early stages of coronary artery changes, and are most predictive of later disease in preclinical populations [15,18-20].

HRV arises from a combination of sources. Frequency domain analyses, which use various forms of spectral decomposition or autoregressive techniques, have divided HRV into components defined by the frequencies at which fast and slow periods of relative heart rate occur. Two components, which primarily reflect the autonomic control of sinoatrial node firing, are conventionally known 
as high frequency (HF; $0.15-0.4 \mathrm{~Hz}$;) and low frequency (LF; $0.05-0.15 \mathrm{~Hz}$ ) HRV [21]. The HF component is associated with the respiratory rhythm (respiratory sinus arrhythmia), primarily regulated by parasympathetic, vagal influences on cardiac rhythm. Some researchers have proffered LF power as an index of sympathetic modulation of heart rate, whereas others contest this interpretation because the effects of experimental sympathetic and parasympathetic stimulation or blockade do not always produce the effects on LF power that sympathetic mediation would predict [21]. The LF is associated with synchronous fluctuations in blood pressure (BP) and baroreflex activity [22]. It has therefore been suggested that LF reflects vasomotor activity indirectly, mediated by vagal efferents to the heart, and is best viewed as reflecting sympatho-vagal balance $[23,24]$. For example, in a landmark paper, Akselrod and colleagues [25] demonstrated by combining vagal and $\beta$-sympathetic blockade that LF power was enhanced by sympathetic activation, and also by vagal activation. The concept of sympatho-vagal balance has also been operationalized as the ratio of $\mathrm{LF}$ and $\mathrm{HF}$ power. As atherosclerosis involves structural changes in the vessels that significantly alter peripheral reflexes influencing BP and heart rate regulation [26], LF power may also be altered by the disease state rather than being antecedent to the development of thickened IMT and plaque.

In the current analyses, we investigated the relationship of HRV and carotid IMT in individuals at increased risk for CAD. The Pittsburgh study selected participants with moderate untreated hypertension and no other known cardiovascular disease as well as comparably aged healthy controls. The significant relationship between LF and carotid IMT in this sample led us to perform a parallel analysis on a subsample from Kuopio Ischemic Heart Disease Risk Factor study (KIHD), drawn to compare those with CAD symptoms and healthy controls. This analysis cross-validated our initial observation, that is, showed that the finding could be replicated, and further generalized the result to participants selected on the basis of a related but not identical cardiovascular disease symptom.

\section{Methods \\ Pittsburgh study \\ Participants}

Participants were $40-70$ years old and enrolled in a study conducted at the University of Pittsburgh to identify the psychosocial and biological correlates of carotid artery atherosclerosis in Caucasian and African-American individuals with normal $\mathrm{BP}$ or unmedicated hypertension. As the primary focus of this trial was on the physiology of the autonomic nervous system and its relation to hypertension, the study sought to eliminate the effect of prior and current medications that might alter normal auto- nomic function. Participants were recruited via mass mailings to the residents of communities of Southwestern Pennsylvania (principally Allegheny County). Exclusion criteria included the use of cardiovascular medication in the period 2 months before enrollment; hypertension treatment of more than 12 months within the preceding 5 years or lifetime antihypertensive treatment exceeding 2 years; and secondary hypertension. Additional exclusions included: angina pectoris; myocardial infarction or angioplasty in the preceding 12 months; congestive heart failure, valvular heart disease, atrial fibrillation, renal insufficiency, suspected secondary hypertension, stroke, neurological disorders, pulmonary disease, clinically apparent CAD, or cancer; use of any psychotropic or glucocorticoid medications; and coronary bypass or carotid or peripheral vascular surgery. Diabetic patients were excluded if reporting peripheral neuropathy, use of insulin, or a fasting serum glucose concentration $>200 \mathrm{mg} / \mathrm{dl}$. Hypertensive status was defined by two seated resting BP measurements averaging $140-180 \mathrm{mmHg}$ systolic BP and/or $90-110 \mathrm{mmHg}$ diastolic BP on each of the two screening sessions. Normotensive status was confirmed by similarly assessed BP measurements $<130 \mathrm{mmHg}$ systolic and $<85 \mathrm{mmHg}$ diastolic. The study protocol was approved by the University of Pittsburgh Biomedical Institutional Review Board. An informed, written consent was obtained from all study participants. Table 1 provides further characteristics of the 350 individuals participating in the study.

\section{Design}

Carotid measurements were collected from reclining participants at rest and technicians adjusted the Doppler probe as necessary to obtain a sequence of cardiac cycles from which arterial wall thickness could be accurately assessed. Cardiovascular measures were collected during a separate session within a few days of the carotid testing. This session tested physiological reactivity to stress challenges. Physiological measures, including heart rate and respiratory measures, were collected continuously during 6-min resting baselines that preceded the performance of six cognitive challenge tasks. Given our interest in assessing typical resting state, we chose to assess HRV during a baseline from the middle of the testing session. Baselines yielded comparable BP measures, but the baseline from the middle of the session was judged to be less influenced by the novelty of the testing procedure and fatigue owing to performance in the challenge tasks. See procedures in Manuck et al. [27].

\section{Physiological measurements and processing}

Electrocardiograms were collected from electrodes placed in a modified lead 2 position and the analog signal digitized at a rate of $1000 \mathrm{~Hz}$. Interbeat intervals, the times between sequential heart beats, were subsequently determined by a program that detected the r-wave from the electrocardiogram and assessed the time between 
Table 1 Demographic characteristics and mean intimal-medial thickness (IMT) for the control and disease-related samples in the Pittsburgh sample $(n=351)$ and in the Kuopio Ischemic Heart Disease Risk Factor study sample $(n=63)$

\begin{tabular}{lcc}
\hline & $\begin{array}{c}\text { Normotensive }(n=148) \\
\text { Hypertensive }(n=203)\end{array}$ & $\begin{array}{c}\text { Healthy }(n=36) \\
\text { Angina }(n=27)\end{array}$ \\
\hline Gender (proportion) & 271 Men $(0.77)$ & 63 Men $(1.00)$ \\
& 80 Women $(0.23)$ & 0 Women $(0.00)$ \\
Age mean (SD) & $56.8(8.5)$ & $57.6(2.7)$ \\
Race (proportion) & 217 White $(0.62)$ & 63 White $(1.00)$ \\
& 129 Black (0.36) & \\
Mean arterial pressure (mmHg) & 5 Other (0.02) & \\
Total cholesterol (mg/dl) & $112(13)$ & $96(10)$ \\
Body mass index & $214(39)$ & $227(44)$ \\
Proportion of nonsmokers & $27.8(3.6)$ & $27.0(2.8)$ \\
Total power (ms $\left.{ }^{2}\right)$ & 0.37 & 0.21 \\
Low-frequency power (ms $\left.{ }^{2}\right)$ & $53797(77430)$ & $49720(76648)$ \\
High-frequency power (ms $\left.{ }^{2}\right)$ & $7377(9400)$ & $6820(7436)$ \\
Log of normalized low-frequency & $5610(12492)$ & $2310(4364)^{\mathrm{a}}$ \\
$\quad$ power & $-2.098(0.904)$ & $-2.104(0.594)$ \\
Log of normalized high-frequency & $-2.649(1.699)$ & $-3.404(0.775)$ \\
$\quad$ power & & \\
Mean heart rate (beats per & $71(9)$ & $64(8)$ \\
$\quad$ minute) & & \\
IMT mean in mm (SD) & $0.88(0.15)$ & $1.28(0.27)$ \\
Plaque index & $1.52(1.99)$ & - \\
\hline
\end{tabular}

${ }^{\mathrm{a}}$ For the $0.15-0.50 \mathrm{~Hz}$ bandwidth, mean power was 3578 with a standard deviation of 14848 .

r-waves with millisecond accuracy. The distribution of these intervals and the electrocardiogram with detections indicated were displayed on a computer screen. Trained technicians checked these data for physiological validity and corrected the automated scoring as necessary. Participants with more than three cardiac arrhythmia's (primarily premature ventricular contractions) per minute or nine per total measurement period were omitted from the sample. Owing to the undue influence of arrhythmic beats on spectral output, all premature beats in participants not excluded were edited by splitting the summed adjacent short and long beats and into equal length beats.

Respiration data were collected at $1000 \mathrm{~Hz}$ from a pneumatic belt placed mid-thorax on the volunteers. A Statham pressure gauge sensed pressure within the belt related to respiratory inspiration and expiration. Stored signals were visually scored for respiratory rate and this rate was used to determine the respiratory/HF band for that individual. Movement artifact periods were omitted when determining respiratory rate; records without a total of $2 \mathrm{~min}$ of artifact-free respiration data (less than 5\%) were assigned the mean respiration rate of the rest of the sample (16 breaths per min or $0.27 \mathrm{~Hz}$ with a standard deviation across participants of $0.05 \mathrm{~Hz}$ ).

Carotid artery plaque and intima-media wall thickness measurements were obtained on a separate occasion (typically during the same week) by duplex (B-mode) ultrasonography performed under the direction of Dr Kim Sutton-Tyrell. A Toshiba SSA-270A duplex scanner
Toshiba America, Inc., New York, New York, USA) was used, and scans were recorded on tape for later scoring. Trained sonographers obtained detailed B-mode images of the right and left common carotid artery, carotid bifurcation and the first centimeter of the internal carotid. The sonographer focused on the interfaces required to measure IMT, and the largest area of plaque, if one was present. Scans were scored by trained readers who evaluated the video images for the number and size of atherosclerotic plaques, and the thickness of the intima and media layers of the arterial wall. IMT was measured on the near and far walls of the distal common $(1 \mathrm{~cm}$ proximal to the carotid bulb), the far wall of the carotid bulb (the point where the near and far walls of the common are no longer parallel to the flow divider), and the far wall of the internal carotid (from the flow divider to $1 \mathrm{~cm}$ distal to this point). Advential-medial and intimalumen interfaces were identified for each segment and a measurement was generated for each pixel for an average of 140 measures in each segment. To evaluate the reproducibility of IMT measurements, data from 34 carotid bifurcations were analyzed on two occasions. Intraclass correlation coefficients were 0.95 for distal common, 0.93 for the bulb, and 0.97 for the internal carotid. A plaque score was based on a focal area whose thickness exceeded that in the adjacent segment by more than $50 \%$. Each location on the artery was then scored as 0 , no plaque; 1 , one small plaque; 2 , multiple small or a medium plaque; or 3 , large plaque or multiple medium/ small plaques. These scores were then summed across all artery segments to create the plaque index.

\section{Kuopio Ischemic Heart Disease Risk Factor study}

We tested the replicability and generality of the findings from the Pittsburgh study by performing a parallel analysis in another sample of individuals selected to vary in risk for vascular disease. Archival data from the KIHD study were available for a subset of the individuals participating in a cardiovascular reactivity session as part of the second wave of data collection for this project [28]. KIHD is a community study in Kuopio, Finland, organized by Jukka Salonen [29,30]. All men born in the region in the following four birth years were invited to participate: 1926-1927, 1932-1933, 1938-1939, and 1944-1945. A total of 2682 men (82.9\% of those eligible) participated in the baseline examination. The current data are from a cohort of 1038 KIHD participants who completed a 4-year follow-up re-evaluation between March 1991 and December 1993. Of these men, 902 participated in the cardiovascular reactivity testing, and archival digital tape recordings of these data were available on approximately half of the men. Carotid IMT was assessed during the same 4-year follow-up reevaluation. Those with taped cardiovascular reactivity data, whose responses on the Rose Questionnaire [31] were indicative of angina $(n=33)$, were selected for study on the basis of the assumption that vascular disease 
would be more prevalent. Individuals reporting angina, but also reporting confounding chronic disease and medication use, were excluded from the angina sample. A sample of 39 men with the same ages and availability of tape recordings, was then selected using the criteria of the absence of either angina or any report of chronic disease or medication use. The tape recordings of these 72 men were then processed to recover the electrocardiogram and apply the same analytic procedures as were done for the Pittsburgh study. Technical difficulties and poor initial recordings reduced the sample to $27 \mathrm{men}$ with reported angina and 36 healthy men for comparison. Table 1 shows their characteristics.

Physiological data collection in KIHD was similar to that in the Pittsburgh study. The ultrasound data collection was very similar to that described for the Pittsburgh study, but only the common carotid wall was imaged. Imaging was performed with a Bio-sound Phase 2 scanner equipped with a $10-\mathrm{MHz}$ annular array probe [32]. The stress-reactivity and baseline sessions were essentially identical to those of the Pittsburgh study and the same mid-session baseline was assessed for HRV. Respiratory rate was, however, unavailable and a standard respiratory band at $0.25-0.30 \mathrm{~Hz}$ was defined as the HF band for purposes of HRV assessment. This band encompassed the mean respiratory rate of the Pittsburgh study and was of comparable bandwidth, but a wider band $(0.15-0.50 \mathrm{~Hz})$ was also examined to see whether bandwidth altered the results.

\section{Statistical analyses}

For both data sets, the sequential cardiac interbeat interval time series from the selected resting baseline was assessed to determine its component frequencies using a point process analysis developed at the University of Amsterdam, PSPAT [33]. This program yields results similar to a Fourier decomposition, but does not assume a continuous underlying generator function. Conceptually, the analysis is consistent with the integral-pulsefrequency-modulation approach used in recent modeling of the neural basis of HRV [34]. Total power, power at the individual's mean respiratory frequency $\pm 0.015 \mathrm{~Hz}$ for the Pittsburgh sample or at $0.25-0.30 \mathrm{~Hz}$ and $0.15-0.50$ for the KIHD sample (HF band power), and power at 0.1 $\pm 0.015 \mathrm{~Hz}$ (LF band power) were calculated. The \pm 0.015 bands were chosen on the basis of our prior experience suggesting that respiratory-specific variation as well as LF variability were encompassed by this bandwidth. We employed as dependent variables the total power and the normalized LF and HF power. As noted in the Task Force guidelines [35], expressing LF and $\mathrm{HF}$ as a proportion of total power (normalizing) better represents the relative contribution of power in these bands to the control of HRV. This is particularly true when substantial variation in total power between participants, which characterized this sample, may obscure the relative contribution of vagal and sympathetic influences. Following Task Force recommendations, however, Table 2 also presents the power values in their original units. Owing to the skewness of distribution of these scores, the natural logarithm of the scores was used as the dependent variable in statistical tests in order to better meet the assumptions of parametric analyses. The same variables were derived in the KIHD sample.

Initial bivariate correlations showed that among the physiological measures collected during the rest period, only mean interbeat interval, that is, resting heart rate, and baseline BP were related to the HRV measurements. Mean arterial pressure was controlled by entering it with other risk factors in regression analyses, and mean interbeat interval was entered along with each power into analyses.

Carotid artery measurements were selected to maximize comparability between the samples. The mean IMT was calculated as the mean of all measures from the right and left common carotid arteries. This measure has been shown to index the atherosclerotic process in the arteries and relate to cardiovascular disease outcomes [17,36]. Maximum IMT measures were available in both samples, but mean and maximum IMT were highly correlated, for example $r=0.96$ in the Pittsburgh sample, and yielded essentially similar relationships, thus we present only the mean IMT results. A plaque index was available from the Pittsburgh sample, but a comparably calculated index was not available from KIHD.

\begin{tabular}{|c|c|c|c|c|}
\hline Predictors & $\beta$ & $t$ & $R^{2}$ & Incremental $R^{2}$ \\
\hline \multicolumn{3}{|c|}{ Standard risk factors } & \multicolumn{2}{|l|}{$0.320 * *$} \\
\hline Age & 0.442 & $8.90 * *$ & & \\
\hline Education & -0.125 & $-2.54 *$ & & \\
\hline BMI & 0.083 & 1.71 & & \\
\hline Cholesterol & 0.227 & $4.78 * *$ & & \\
\hline Smoking & 0.003 & 0.06 & & \\
\hline MAP & 0.200 & $3.91 * *$ & & \\
\hline Race & 0.088 & 1.64 & & \\
\hline Sex & -0.178 & $-3.38 * *$ & & \\
\hline \multicolumn{3}{|c|}{ Low-frequency heart rate variability } & $0.333 * *$ & $+0.012 *$ \\
\hline IBI & -0.004 & -0.08 & & \\
\hline LF & -0.120 & $-2.36 * *$ & & \\
\hline \multicolumn{3}{|c|}{ High-frequency heart rate variability } & $0.321^{* *}$ & +0.001 \\
\hline IBI & 0.028 & 0.58 & & \\
\hline $\mathrm{HF}$ & 0.009 & 0.19 & & \\
\hline \multicolumn{3}{|c|}{ Total power heart rate variability } & $0.321 * *$ & +0.001 \\
\hline $\mathrm{IBI}$ & 0.029 & 0.62 & & \\
\hline Total power & 0.006 & 0.13 & & \\
\hline
\end{tabular}

Note: Standard cardiovascular risk factors are entered initially. Baseline cardiac interbeat interval and power estimates are added separately to see whether a significant increment in predictivity occurs ( $N$ of 350 , reduced to 332 owing to missing data on one or more predictors).

$\mathrm{BMI}$, body mass index; MAP, mean arterial pressure; IBI, inter-beat-interval; LF, low frequency; HF, high frequency.

$* P<0.05, * * P<0.01$. 
Regression analyses (Statistica, StatSoft Inc., Tulsa, Oklahoma City, USA) were used to ask three questions. First, whether LF, HF, or total HRV was related to mean IMT. Second, whether HRV indices accounted for any IMT variability beyond that associated with standard risk factors for atherosclerosis or with average heart rate. Separate analyses were performed within hypertensive and normotensive groups for the Pittsburgh sample and within angina and comparison groups for the Kuopio sample. Comparable results were routinely obtained, suggesting that the relationships to be reported were not modulated by the presence of either hypertension or angina.

\section{Results}

Table 1 presents the demographic and descriptive variables for the two samples. The samples are similar in average age but differ notably in sex composition, racial composition, and sampling criteria (hypertension and angina).

\section{Pittsburgh sample}

In simple bivariate analyses, mean carotid IMT was found to correlate inversely with $\mathrm{LF}$ power $(r=-0.19$, $P<0.001)$ and total power $(r=-0.16, \quad P<0.01)$, whereas no significant relationship existed between $\mathrm{HF}$ power or the ratio of $\mathrm{LF} / \mathrm{HF}$ power and IMT. The plaque index was similarly correlated with LF power $(r=-0.13$, $P<0.05)$ and total power $(r=-0.16, P<0.01)$.

We then asked whether HRV accounted for any variance in IMT or plaque index beyond that associated with standard risk factors for atherosclerosis and baseline heart rate (mean interbeat interval). In this stepwise analysis, we first entered the basic risk factors for atherosclerotic disease (age, race, sex, education, body mass index, cholesterol, smoking, and mean arterial pressure), and in the second step we separately assessed LF, HF, and total power. Power estimates were entered along with mean interbeat interval to see whether power contributed to variance in carotid IMT independently of the simpler measure of autonomic control. Table 2 provides the results of these analyses. The Pittsburgh sample showed that standard risk factors explained a large portion of the variance in IMT. Nonetheless, in this regression model, lower LF power still contributed independently as a predictor of greater wall thickening. Figure 1 illustrates the relationship by plotting quartiles of LF power versus mean IMT. In parallel analyses, no relationship was evident between total power or HF power and IMT or between any of the HRV measures and the plaque index, once standard risk factors were entered. Given recent standards for glucose levels defining diabetes, all analyses were rerun including only participants with glucose $<125 \mathrm{mg} / \mathrm{dl} \quad(n=319)$. All significant effects remained significant and in this group LF power
Fig. 1

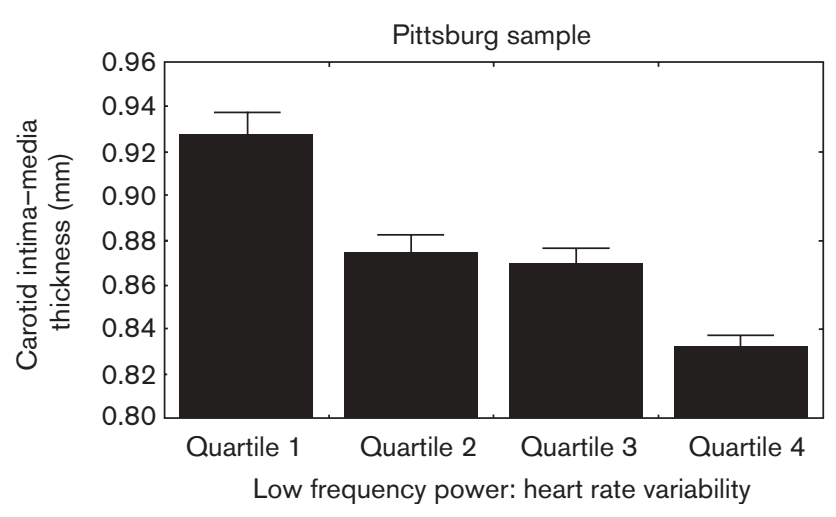

Mean carotid IMT as a function of quartile of power in the low frequency heart rate variability band for the Pittsburg sample. Quartile 1 is lowest amount of variability; Quartile 4 the highest.

was now significantly associated with the plaque index $(\beta=-0.116, t=-2.06, P<0.05)$ with standard risk factors controlled.

\section{Kuopio Ischemic Heart Disease Risk Factor study sample}

As LF power showed statistically significant but not substantial relations in the Pittsburgh study, replication of this result was important. Replication in a separate sample, similarly at risk for atherosclerotic disease, would provide strong evidence of the generalizability of the results and support the validity of the statistical evidence of this from the Pittsburgh sample.

Within the KIHD sample, simple bivariate analyses showed again that LF power was found to correlate inversely with mean carotid IMT $(r=-0.23, P=0.06)$ as did total power $(r=-0.24, P<0.05)$, whereas no significant relationship existed between HF power (at both $0.25-0.30$ and $0.15-0.50 \mathrm{~Hz}$ bandwidths) or a ratio of LF/HF power and IMT. Given these results, a hierarchical regression analysis parallel to that of the Pittsburgh study was performed.

Table 3 provides the findings of regression analyses of the KIDH sample and specifically shows that LF power was again a significant predictor independent of standard risk factors. The standard cardiovascular risk factors entered as step 1 were not significantly predictive of IMT in this small subsample (but see results for whole sample) [30]. When baseline heart rate and LF were entered, LF was independently predictive of IMT $(t=-2.20, P<0.05)$, but the overall model was not significant $\left(R^{2}=0.172\right.$, NS). Figure 2 illustrates the relationship between LF power and carotid IMT. Again in 
Table 3 Hierarchical regression analysis for Kuopio Ischemic Heart Disease Risk Factor study subsample $(n=63)$

\begin{tabular}{|c|c|c|c|c|}
\hline Predictors & $\beta$ & $t$ & $R^{2}$ & Incremental $R^{2}$ \\
\hline \multicolumn{3}{|c|}{ Standard risk factors } & \multicolumn{2}{|l|}{0.098} \\
\hline Age & -0.047 & -0.36 & & \\
\hline Education & -0.031 & -0.23 & & \\
\hline BMI & 0.124 & 0.86 & & \\
\hline Cholesterol & 0.145 & 0.98 & & \\
\hline Smoking & 0.240 & 1.80 & & \\
\hline MAP & -0.033 & -0.24 & & \\
\hline \multicolumn{3}{|c|}{ Low-frequency heart rate variability } & 0.172 & \multirow[t]{3}{*}{+0.086} \\
\hline IBI & -0.166 & -1.01 & & \\
\hline LF & -0.372 & $-2.20 *$ & & \\
\hline \multicolumn{3}{|c|}{ High-frequency heart rate variability ${ }^{\mathrm{a}}$} & 0.101 & \multirow[t]{3}{*}{+0.003} \\
\hline IBI & 0.042 & 0.30 & & \\
\hline $\mathrm{HF}$ & 0.045 & 0.34 & & \\
\hline \multicolumn{3}{|c|}{ Total power heart rate variability, with IBI } & 0.186 & \multirow[t]{3}{*}{+0.074} \\
\hline $\mid \mathrm{BI}$ & -0.019 & -0.13 & & \\
\hline Total Power & -0.233 & -1.62 & & \\
\hline
\end{tabular}

Note: Standard cardiovascular risk factors are entered initially. Baseline cardiac interbeat interval and power estimates are added separately to see whether a significant increment in predictivity occurs.

$\mathrm{BMI}$, body mass index; MAP, mean arterial pressure; IBI, inter-beat-interval; LF, low frequency; HF, high frequency.

${ }^{a}$ Values presented for $0.25-0.30 \mathrm{~Hz}$. For the $0.15-0.50 \mathrm{~Hz}$ bandwidth, IBI had a $\beta$ and $t$ value of -0.05 and -0.36 , and HF a $\beta$ and $t$ value of 0.15 and 1.18 . These as well as the incremental $R^{2}$ of 0.02 were statistically nonsignificant. $* P<0.05, * * P<0.01$

this sample, neither HF nor total power was significantly related to IMT.

\section{Discussion}

A pattern of correlation of lower LF power with greater carotid IMT was evident in the larger Pittsburgh sample, and repeated in the smaller KIHD subsample. In light of the substantial differences between KIHD and Pittsburgh samples, as well as the less marked methodological differences, the replication of the correlation between LF power and IMT is suggestive of a robust, albeit modest, relationship between $\mathrm{LF}$ and a putative index of coronary atherosclerosis. The strength of the relationship was similar to the strength of the relationship between total cholesterol and carotid IMT in this sample $(r=0.21$ for cholesterol, $r=-0.19$ for LF power). Furthermore, LF power was predictive independently of the heart rate and the standard risk factors we assessed (including resting $\mathrm{BP})$. The latter may be particularly important in that carotid IMT is likely influenced both by atherosclerotic processes and by vascular remodeling related to $\mathrm{BP}$ $[37,38]$.

Reductions in total power, LF, and HF power have been related to cardiovascular disease $[39,40]$. HF power can be interpreted in terms of vagal influence on the heart, but LF power, not HF power, was related to carotid IMT in the current samples. Neural and vascular wall mechanisms may be relevant to the relation of reduced LF power and greater carotid IMT.
Fig. 2

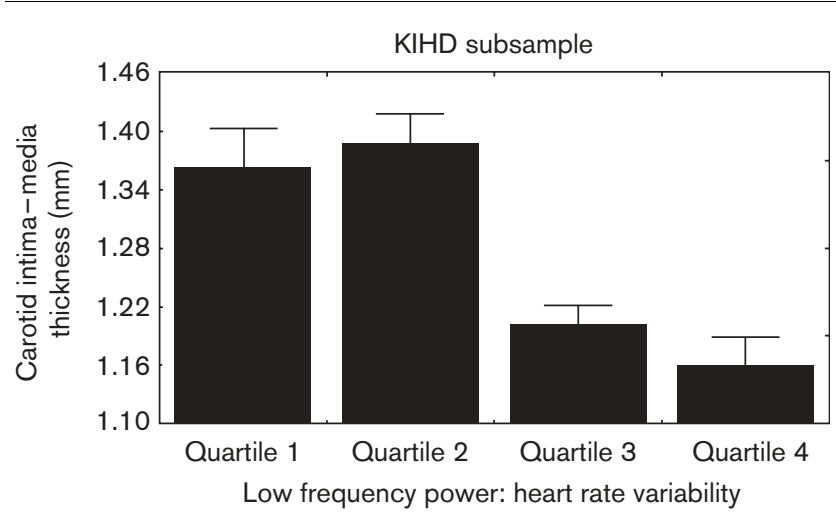

Carotid mean IMT as a function of power in the low frequency heart rate variability band for the KIHD subsample. Quartile 1 is the lowest amount of variability; Quartile 4 is the highest.

Considering neural mechanisms first, LF power is known to reflect directly sympathetic and parasympathetic influences on the heart. The observed association between carotid artery IMT with reduced LF, but not HF power, might then suggest a relationship between atherosclerosis and reduced sympathetic nervous system activity $[25,35]$. This interpretation tends to contradict the body of evidence suggesting that heightened, not damped, sympathetic activation relates to cardiovascular disease [41]. Even in the current studies, other evidence links heightened sympathetic reactivity and carotid IMT [42-44]. We could speculate that chronic sympathetic overactivity might downregulate vascular sympathetic receptors over time, leading to the observed relationship between low LF power and carotid IMT.

Alternatively, vascular wall characteristics may influence LF power and these may interact with autonomic function. The stiffening of vessel walls and impaired vascular endothelial function found in atherosclerosis may have implications for LF power. Rhythmic vascular contractions will alter baroreceptor output and subsequently HRV. Changes in vessel walls may diminish both the amplitude and regularity of vessel wall contractions, and also the sensitivity of the baroreceptors. Reduced baroreceptor stimulation could be due to stiffening of the vessel wall that surrounds the receptors $[45,46]$. Decreased baroreceptor gain was related to greater carotid IMT in a Pittsburgh sample similar to the current one [47]. Colhoun et al. [12] described an alternative mechanism involving the reverse causal relationship, in which autonomic dysfunction may contribute to the pathogenesis of atherosclerosis. They review evidence that sympathetic denervation may cause vascular smooth muscle cells to dedifferentiate, resulting in a cell phenotype associated with intimal migration and extracellular matrix production, characteristic of atherosclero- 
sis. Such a scenario may relate to the suggestion of Sloan et al. [48] that low HRV may be due to high and unregulated BP variability. They further suggest that high BP variability contributes to the development of coronary atherosclerosis. In short, the current observations may relate either to changes in autonomic control that contribute to atherosclerosis or to atherosclerotic change that alters HRV. Prospective and experimental studies will be required to resolve the direction of causation.

\section{Limitations}

Our results are limited by their cross-sectional nature and the sampling involved. Both of our samples were selected for disease status rather than random sampling. As such, they can demonstrate the likelihood of a relationship between HRV and carotid atherosclerosis, but not its generalizability to unselected populations. Our HRV measures would ideally have been informed by the assessment of respiratory volume and frequency in the Pittsburgh sample and by the measurement of both in the KIHD sample. Within the KIHD sample, we adopted a relatively narrow respiratory bandwidth on the basis of the average of the Pittsburgh sample. This provided statistical comparability, but may have missed substantial HF variability in individuals breathing outside of this range. Assessment of a wider band, $0.15-0.50 \mathrm{~Hz}$, showed the same pattern of results, however. Details of the carotid assessment also differed between samples and comparable plaque indices were unavailable.

\section{Conclusion}

Similar findings of lower LF power and atherosclerotic vessel changes in individuals who do not have advanced CAD suggests that LF may be useful in increasing the predictive accuracy of coronary artery progression. Although relationships of LF HRV and pathogenesis are complex, a prime contributor is likely to be diminished vascular wall distensibility, resulting in decreased baroreflex influence on heart rate. Prospective studies making use of increasingly well identified markers for risk of CAD are needed to further explore pathogenic relationships among autonomic function, HRV, and atherosclerosis.

\section{Acknowledgements}

This work was supported by grants from the Academy of Finland and the Finnish Ministry of Education and the National Heart, Lung, and Blood Institute (HL-44199 and HL-40962).

\section{References}

1 Wolf MM, Varigos GA, Hunt D, Sloman JG. Sinus arrhythmia in acute myocardial infarction. Med J Aust 1978; 2:52-53.

2 van Ravenswaaij-Arts CMA, Kollee LAA, Hopman JCW, Stoelinga GBA, van Geijin HP. Heart rate variability. Ann Intern Med 1993; 118:436-447.

3 Galinier M, Pathak A, Fourcade J, Androdias C, Curnier D, Varnous S, et al. Depressed low frequency power of heart rate variability as an independent predictor of sudden death in chronic heart failure. Eur Heart J 2000; 21:475-482.
4 Kleiger RE, Miller JP, Bigger JT Jr, Moss AJ; the Multicenter Post-Infarction Research Group. Decreased heart rate variability and its association with increased mortality after acute myocardial infarction. Am J Cardiol 1987; 59:256-262.

5 Tsuji H, Larson MG, Venditti FJ Jr, Manders ES, Evans JC, Feldman CL, Levy D. Impact of reduced heart rate variability on risk for cardiac events. The Framingham Heart Study. Circulation 1996; 94:2850-2855.

6 Bigger TJ, Fleiss JL, Steinman RC, Rolnitzky LM, Kleiger RE, Rottman JN. Frequency domain measures of heart period variability and mortality after myocardial infarction. Circulation 1992; 85:164-171.

7 Dekker JM, Crow RS, Folsom AR, Hannan PJ, Liao D, Swenne CA, Schouten EG. Low heart rate variability in a 2-min rhythm strip predicts risk of coronary heart disease and mortality from several causes: the ARIC Study. Atherosclerosis Risk In Communities. Circulation 2000; 102:1239-1244.

8 Kupari M, Virolainen J, Koskinen P, Tikkanen M. Short-term heart rate variability and factors modifying the risk of coronary artery disease in a population sample. Am J Cardiol 1993; 72:897-903.

9 Huikuri HV, Jokinen V, Syvanne M, Nieminen MS, Airaksinen KE, Ikaheimo $\mathrm{MJ}$, et al. Heart rate variability and progression of coronary atherosclerosis. Arterioscler Thromb Vasc Biol 1999; 19:1979-1985.

10 Hayano J, Sakakibara Y, Yamada M, Ohte N, Fujinami T, Yokoyama K, et al. Decreased magnitude of heart rate spectral components in coronary artery disease: its relation to angiographic severity. Circulation 1990; 81: 1217-1224.

11 Liao D, Cai J, Rosamond WD, Barnes RW, Hutchinson RG, Whitsel EA, et al. Cardiac autonomic function and incident coronary heart disease: a population-based case-cohort study. The ARIC Study. Atherosclerosis Risk in Communities Study. Am J Epidemiol 1997; 145:696-706.

12 Colhoun HM, Francis DP, Rubens MB, Underwood SR, Fuller JH. The association of heart-rate variability with cardiovascular risk factors and coronary artery calcification: a study in type 1 diabetic patients and the general population. Diabetes Care 2001; 24:1108-1114.

13 Mack WJ, LaBree L, Liu C, Selzer RH, Hodis HN. Correlations between measures of atherosclerosis change using carotid ultrasonography and coronary angiography. Atherosclerosis 2000; 150:371-379.

14 Adams MR, Nakagomi A, Keech A, Robinson J, McCredie R, Bailey BP, et al. Carotid intima-media thickness is only weakly correlated with the extent and severity of coronary artery disease. Circulation 1995; 92:2127-2134.

15 Chambless LE, Heiss G, Folsom AR, Rosamond W, Szklo M, Sharrett AR, Clegg LX. Association of coronary heart disease incidence with carotid arterial wall thickness and major risk factors: the Atherosclerosis Risk in Communities (ARIC) Study, 1987-1993. Am J Epidemiol 1997; 146:483-494.

16 O'Leary DH, Polak JF, Wolfson SK, Bond MG, Bommer W, Sheth S, et al. Use of sonography to evaluate carotid atherosclerosis in the elderly: The Cardiovascular Health Study. Stroke 1991; 22:1155-1163.

17 O'Leary DH, Polak JF, Kronmal RA, Manolio TA, Burke GL, Wolfson SK Jr. Carotid-artery intima and media thickness as a fist factor for myocardial infarction and stroke in older adults. Cardiovascular Health Study Collaborative Research Group. N Engl J Med 1999; 340:14-22.

18 Rothwell PM. Carotid artery disease and the risk of ischaemic stroke and coronary vascular events. Cerebrovasc Dis 2000; 10:21-33.

19 Polak JF, Kronmal RA, Tell GS, O'Leary DH, Savage PJ, Gardin JM, et al. Compensatory increase in common carotid artery diameter: relation to blood pressure and artery intima-media thickness in older adults. Stroke 1996; 27:2012-2015.

20 Sidhu PS, Allan PL. The extended role of carotid artery ultrasound. Clin Radiol 1997; 52:643-653.

21 Berntson GG, Bigger JT, Eckberg DL, Grossman P, Kaufmann PG, Malik M, et al. Heart rate variability: origins, methods, and interpretive caveats. Psychophysiology 1997; 34:623-648.

22 Kamath MV, Ghista DN, Fallen EL. Heart rate variability power spectrum as a potential noninvasive signature of cardiac regulatory response, mechanisms, and disorders. Heart Vessels 1987; 3:33-41.

23 Akselrod S, Gordon D, Madwed JB, Snidman NC, Shannon DC, Cohen RJ. Hemodynamic regulation: investigation by spectral analysis. Am J Physiol 1985; 18:H867-H875.

24 Pomeranz B, Macaulay RJB, Caudill MA, Kutz I, Adam D, Gordon D, et al. Assessment of autonomic function in humans by heart rate spectral analysis. Am J Physiol 1985; 248:H151-H153.

25 Akselrod S, Gordon D, Ubel FA, Shannon DC, Berger AC, Cohen RJ. Power spectrum analysis of heart rate fluctuation: a quantitative probe of beat-to-beat cardiovascular control. Science 1981; 4504:220-222.

26 Heistad DD, Baumbach FM, Faraci FM, Armstrong ML. Sick vessel syndrome: vascular changes in hypertension and atherosclerosis. J Hum Hypertens 1995; 9:449-453. 
27 Manuck SB, Polefrone JM, Terrell DF, Muldoon MF, Kasprowicz AL, Waldstein SR, et al. Absence of enhanced sympathoadrenal activity and behaviorally evoked cardiovascular reactivity among offspring of hypertensives. Am J Hypertens 1996; 9:248-255.

28 Jennings JR, Kamarck T, Manuck S, Everson SA, Kaplan G, Salonen JT. Aging or disease? Cardiovascular reactivity in Finnish men over the middle years. Psychol Aging 1997; 12:225-238.

29 Salonen JT. Is there a continuing need for longitudinal epidemiological research? The Kuopio Ischemic Heart Disease Risk Factor Study. Ann Clin Res 1998; 20:46-50.

30 Salonen JT, Salonen R. Risk-factors for carotid and femoral atherosclerosis in hypercholesterolemic men. Ann Intern Med 1994; 236:561-566.

31 Rose G, McCartney P, Reid DD. Self-administration of a questionnaire on chest pain and intermittent claudication. Br J Prevent Soc Med 1977; 31:42-48.

32 Salonen JT, Salonen R. Intima-media changes in a population study: KIHD In: Boccalon J, editor. Vascular medicine. Amsterdam: Elsevier Science; 1993. pp. 301-304.

33 Weber EJM, Molenaar PCM, vanderMolen MW. PSPAT: a program for spectral analysis of point events including a test for stationarity. In: Mulder LM, Maarse FJ, et al. editors. Computers in psychology: Applications in education, research and psychodiagnostics. Lisse, Netherlands: Swets \& Zeitlinger; 1991. pp. 132-139.

34 Pyetan E, Akselrod S. Do the high-frequency indexes of HRV provide a faithful assessment of cardiac vagal tone? A critical theoretical evaluation. IEEE Trans Biomed Eng 2003; 50:777-783.

35 Task Force. Heart rate variability: standards of measurement, physiological interpretation, and clinical use. Circulation 1996; 93:1054-1065.

36 Wofford JL, Kahl FR, Howard GR, McKinney WM, Toole JF, Crouse JR. Relation of extent of extracranial carotid artery atherosclerosis as measured by B-mode ultrasound to the extent of coronary atherosclerosis. Arterioscler Thromb 1991; 11:1786-1794.

37 Al-Shali K, House AJ, Hanley AJ, Khan HM, Harris SB, Mamakeesick M, et al. Differences between carotid wall morphological phenotypes measured by ultrasound in one, two and three dimensions. Atherosclerosis 2005; 178:319-325.
38 Spence JD. Ultrasound measurement of carotid plaque as a surrogate outcome for coronary artery disease. Am J Cardiol 2002; 89 (Suppl): 10B-16B.

39 Stein PK. Inferring vagal tone from heart rate variability. Psychosom Med 1994; 56:577-578.

40 Kleiger RE, Stein PK, Bigger JT. Heart rate variability: measurement and clinical utility. Ann Noninvas Electrocardiol 2005; 10:88-101.

41 Treiber FA, Kamarck T, Schneiderman N, Sheffield D, Kapuku G, Taylor T. Cardiovascular reactivity and development of preclinical and clinical disease states. Psychosom Med 2003; 65:46-62.

42 Kamarck TW, Everson SA, Kaplan GA, Manuck SB, Jennings JR, Salonen R Salonen JT. Exaggerated blood pressure responses during mental stress are associated with enhanced carotid atherosclerosis in middle-aged Finnish men. Circulation 1997; 96:3842-3848.

43 Jennings JR, Kamarck TW, Everson-Rose SA, Kaplan GA, Manuck SB, Salonen JT. Exaggerated blood pressure responses during mental stress are prospectively related to enhanced carotid atherosclerosis in middle-aged Finnish men. Circulation 2004; 110:2198-2203.

44 Gianaros PJ, Bleil ME, Muldoon MF, Jennings JR, Sutton-Tyrrell K, McCaffrey J, Manuck SB. Is cardiovascular reactivity associated with carotid atherosclerosis among hypertensives? Hypertension 2002; 40: 742-747.

45 Jensen-Urstad K, Reichard $\mathrm{P}$, Jensen-Urstad M. Decreased heart rate variability in patients with type 1 diabetes mellitus is related to arterial wall stiffness. J Intern Med 1999; 245:57-61.

46 Tokudome T, Mizushige K, Ohmori K, Watanabe K, Takagi Y, Takano Y, Matsuo $\mathrm{H}$. Neurogenic regulation of basal tone of coronary artery with mild atherosclerosis in humans: observation using two-dimensional intravascular ultrasound. Angiology 1999; 50:989-996.

47 Gianaros PJ, Jennings JR, Olafsson GB, Steptoe A, Sutton-Tyrrell K, Muldoon MF, Manuck SB. Greater intima-media thickness in the carotid bulb is associated with reduced baroreflex sensitivity. Am J Hypertens 2002; 15:486-491

48 Sloan RP, Shapiro PA, Bagiella E, Myers MM, Gorman JM. Cardiac autonomic control buffers pressure variability responses to challenge: a psychophysiologic model of coronary artery disease. Psychosom Med 1999; 61:58-68. 